\title{
A combination of p40, GATA-3 and uroplakin II shows utility in the diagnosis and prognosis of muscle-invasive urothelial carcinoma
}

\author{
Mariah Z. Leivo ${ }^{1}$, Paul J. Elson ${ }^{2}$, David E. Tacha ${ }^{3}$, Brett Delahunt ${ }^{4}$ and \\ DonNa E. HANSEL ${ }^{1}$ \\ ${ }^{1}$ Department of Pathology, University of California San Diego, La Jolla, CA, ${ }^{2}$ Quantitative \\ Health Sciences Institute, Cleveland Clinic, Cleveland, $\mathrm{OH},{ }^{3}$ Biocare Medical, Concord, CA, \\ USA; and ${ }^{4}$ Department of Pathology and Molecular Medicine, Wellington School of Medi- \\ cine and Health Sciences, University of Otago, Wellington South, New Zealand
}

\begin{abstract}
Summary
Recently developed antibodies against uroplakin II and $\Delta$ Np63 (p40) show utility in diagnosing primary bladder urothelial carcinoma, although application in metastatic bladder cancer and patient outcomes has been less well defined. We evaluated these antibodies by immunostain intensity and $\mathrm{H}$-score, in conjunction with GATA-3 immunoreactivity, in 89 patients with muscle-invasive urothelial carcinoma and 35 paired metastasis. The maintenance of immunoreactivity in metastatic lesions and the association to disease recurrence and survival was assessed. Bladder urothelial carcinoma showed immunoreactivity for GATA-3 in $99 \%$ (88/89), p40 in 87\% (77/89) and uroplakin II in 80\% $(71 / 89)$ of cases, with a positive correlation between GATA3 and uroplakin II $\mathrm{H}$-score $(0.44 ; p<0.0001)$. All lesions were positive for at least one marker, reinforcing the use of these markers as a panel. In 35 patients with paired lymph node metastasis, uroplakin II and GATA-3 were retained in $90 \%$ and $75 \%$ of patients, respectively, suggesting these markers may have relatively high sensitivity in diagnosing metastatic urothelial carcinoma. High intensity p40 immunostain (3+), however, was significantly associated with reduced patient survival $(p=0.03)$. These results suggest that whereas GATA-3 and uroplakin II may be most useful in the diagnosis of urothelial carcinoma metastasis, p40 may be additionally suited as a prognostic marker.
\end{abstract}

Key words: Bladder cancer; urothelial carcinoma; uroplakin II; GATA-3; $\mathrm{p} 40 ; \Delta \mathrm{Np} 63$; immunohistochemistry; survival.

Received 3 April, revised 23 May, accepted 24 May 2016 Available online 2 September 2016

\section{INTRODUCTION}

Use of immunohistochemistry (IHC) in the context of urothelial carcinoma (UCa) is primarily required in distinguishing UCa from secondary malignancies involving the urinary tract and in correctly identifying the site of origin for metastasis of unknown primary. Application of IHC markers for assessment of metastatic risk and prognosis in $\mathrm{UCa}$ remains limited. Recently, new antibodies targeting uroplakin II (UPII) and p40 have been developed and show high sensitivity and specificity in the diagnosis of $\mathrm{UCa} ;{ }^{1-7}$ however, few analyses have assessed these markers in combination or in the setting of patient outcomes. In this study, we test the ability of these markers, singly or in combination with GATA-3, to improve diagnostic accuracy and outcomes prediction in advanced UCa.

IHC markers commonly used in the diagnosis of muscleinvasive UCa include uroplakin III (UPIII), GATA-3, thrombomodulin, p63, high-molecular weight cytokeratins and cytokeratin $7 .^{8}$ UPIII shows the highest specificity of this group, but is immunoreactive in only approximately $40-50 \%$ of these cases. ${ }^{9,10}$ Improved sensitivity can be achieved through a combination of IHC markers, although usefulness in the diagnosis of metastatic lesions and variant morphologies tends to be less robust.

In the current study, we assess the ability of UPII, p40 and GATA-3, individually and in combination, to label primary and metastatic UCa and to evaluate the association of these markers and patient prognosis.

\section{MATERIALS AND METHODS}

\section{Patient material}

Patients with invasive UCa were identified through database search at the University of California at San Diego with approval from the Institutional Review Board (Table 1). All cases were re-reviewed by two of the authors (DH and ML). Of the 89 cystectomy cases included for evaluation, pathological stage included pT2 $(19 ; 20 \%)$; pT3 $(62 ; 70 \%)$ and pT4 $(6 ; 7 \%)$ Variant morphology was excluded, including cases with divergent differentiation. Patients included 71 men and 18 women with a mean age of 64 years (range 43-85 years) and a mean follow-up of 25.3 months (range 1-83 months; median 18 months). Estimated median overall survival and recurrence-free survival was 21.5 months [95\% confidence interval (CI) 15.4-29.5] and 18.5 months (95\% CI 13.0-26.9), respectively. In addition, 10 specimens of normal urothelium were included for study and obtained from patients with no prior or subsequent history of UCa.

\section{Immunohistochemistry and scoring}

We performed IHC on formalin fixed, paraffin embedded tissue microarrays (TMA) from normal urothelium (non-cancer patients) and patients with bladder UCa. The TMA were assembled using an automated operated device (TMA Master; 3DHOSTECH Ltd, Hungary) and contained four distinctive $1 \mathrm{~mm}$ diameter cores punched from each case. Four $\mu \mathrm{m}$ sections were cut into 
Table 1 Clinicopathological features

\begin{tabular}{|c|c|}
\hline Features & All patients \\
\hline \multicolumn{2}{|l|}{ Gender } \\
\hline Male & $71 / 89(80 \%)$ \\
\hline Female & $18 / 89(20 \%)$ \\
\hline Age at diagnosis, years (range) & $64(43-85)$ \\
\hline$<75$ & $59 / 89(66 \%)$ \\
\hline$\geq 75$ & $30 / 89(34 \%)$ \\
\hline \multicolumn{2}{|l|}{ Pathological T-stage } \\
\hline 1,2 & $21 / 89(24 \%)$ \\
\hline 3,4 & $68 / 89(76 \%)$ \\
\hline \multicolumn{2}{|l|}{ Carcinoma in situ } \\
\hline No & $42 / 89(47 \%)$ \\
\hline Yes & $47 / 89(53 \%)$ \\
\hline \multicolumn{2}{|l|}{ Positive margins } \\
\hline No & $62 / 89(70 \%)$ \\
\hline Yes & $27 / 89(30 \%)$ \\
\hline \multicolumn{2}{|l|}{ Neoadjuvant or adjuvant chemotherapy } \\
\hline No & $54 / 89(63 \%)$ \\
\hline Yes & $32 / 89(37 \%)$ \\
\hline Overall survival, months, median (range) & $21.5(15.4-29.5)$ \\
\hline Recurrence-free survival, months, median (range) & $18.5(13.0-26.9)$ \\
\hline Follow-up available & $87 / 89(98 \%)$ \\
\hline Length of follow-up, months, median (range) & $18(1-83)$ \\
\hline Patient with recurrent disease & $32 / 87(36 \%)$ \\
\hline Total number of deaths & $55 / 87(62 \%)$ \\
\hline
\end{tabular}

slides and stained with H\&E to verify tumour foci available for optimal IHC evaluation. Additional $4 \mu \mathrm{m}$ sections were cut and deparaffinised in xylene substitute and rehydrated via graded ethanol, followed by blocking of endogenous peroxidases with $3 \%$ hydrogen peroxide solution. Heat-induced antigen retrieval was performed using a modified citrate buffer formulation in a pressure cooker. A standard IHC technique was performed using prediluted mouse monoclonal UPII (clone BC21), GATA3 (clone L50-823), and p40 (clone $\mathrm{BC} 28$ ); all three primary antibodies were purchased from Biocare Medical, USA. All primary antibodies were incubated at room temperature for $30 \mathrm{~min}$. Detection was performed with a rabbit anti-mouse $\mathrm{IgG}$ antibody for $10 \mathrm{~min}$, followed by a horseradish peroxidase polymer conjugate for 10 min. Visualisation was achieved with 3,3'-diaminobenzidine (DAB)

GATA-3 and p40 immunostains were scored for nuclear expression, whereas UPII immunostain was scored for cytoplasmic and membranous staining. Immunohistochemical stain was characterised by intensity $(0$, negative; 1 , weak; 2 , moderate; 3 , strong) with $5 \%$ reactivity used as a cut-off $\mathrm{H}$-score was also used to semiquantify immunostaining using the following formula: $(0 \times \%$ cells 0$)+(1 \times \%$ cells $1+)+(2 \times \%$ cells $2+)+(3 \times \%$ cells $3+)$; resultant scores ranged from 0 to $300 .{ }^{11}$ The immunostains were scored by two of the authors (ML and DH) and discrepant scores were re-evaluated together in order to reach consensus.

\section{Statistical analysis}

The relationship between immunostain intensity (categorical) or H-score (continuous) categories to clinicopathological features was performed using the Fisher's exact test for categorical variables and Wilcoxon rank sum test for continuous variables. Correlation between individual immunohistochemical stains was performed using the Spearman correlation coefficient. Statistical analysis of IHC intensity or H-score relationship to outcomes (disease-specific recurrence and overall survival) was performed using the Logrank test for categorical factors and Wald test from proportional hazards model for continuous factors.

\section{RESULTS}

\section{Immunoreactivity of UPII, GATA-3 and p40 in normal urothelium}

Normal urothelium showed a weakly cytoplasmic to membranous staining pattern of UPII, with reactivity in the intermediate cell layer and more strongly within the umbrella cell layer. GATA-3 was present in the full thickness of the urothelium, whereas p40 was most highly expressed in the basal and intermediate cells of the urothelium (Fig. 1).

\section{GATA-3, UPII and p40 expression in bladder urothelial carcinoma}

We categorised IHC by intensity $(0-3+)$, as well as by $\mathrm{H}-$ score. Bladder UCa showed an overall immunopositivity rate of GATA-3 99\% (88/89), p40 87\% (77/89) and UPII $80 \%$ (71/89) (Table 2). When immunoreactivity was present, the staining pattern was frequently uniform throughout the tumour, with occasional patchy expression in the case of UPII. All cases showed at least one marker to be immunoreactive (Fig. 2). Whereas GATA-3 showed higher intensity stain $(2-3+)$ in $93 \%$ of the specimens, UPII and p40 were less robust at $74 \%$ and $79 \%$ staining level $2-3+$, respectively. No significant association was identified between IHC intensity in the 89 patients and clinicopathological features including gender, age, pathological stage, presence of urothelial carcinoma in situ, and positive margin status. Comparison of the relationship between immunostains showed a positive correlation only between GATA-3 and UPII $(0.44 ; p<0.0001$; Spearman correlation coefficient).

We further subdivided our patient population into those with and without lymph node metastases at the time of cystectomy, which resulted in 43/89 patients (48\%) with metastatic disease and $46 / 89$ patients (52\%) without (Table 2). Pathological features that were more likely in patients with metastatic disease included higher stage (pT3 and pT4 stage; $p=0.01$, Fisher's exact test) and administration of neoadjuvant or adjuvant chemotherapy $(p<0.001$, Fisher's exact test). We also analysed the correlation between $\mathrm{H}$ scores and the presence or absence of lymph node metastasis using Wilcoxon rank sum test; patients with lymph node metastasis showed higher UPII H-score (average 160 versus 90; $p=0.007)$, lower p40 H-score (150 versus 220; $p=0.001)$ and no statistically significant difference in GATA-3 level (250 versus $240 ; p=0.35$ ) in carcinoma cells within the bladder.

\section{UPII is most likely to be retained in metastatic lymph node deposits}

We analysed a subset of 35 patients who had both bladder UCa and matched lymph node metastases to determine if GATA-3, UPII and/or p40 expression is retained in metastatic deposits. Even though a total of 43 patients had documented metastatic disease, only 35 metastatic foci had adequate tumour volume on TMA analysis to accurately score all three immunomarkers. Expression of GATA-3, UPII and p40 (any intensity from $1+$ to $3+$ ) was present in the primary bladder tumour in $35 / 35$ (100\%), 32/35 (91\%) and $28 / 35(80 \%)$ cases, respectively. Comparison to the matched metastatic deposit showed highest retention of UPII expression in the metastatic deposit. When compared to the primary tumour, UPII was retained at similar levels in 29/32 metastatic lesions (90\%), whereas GATA-3 was retained in $26 / 35$ metastases $(74 \%)$ and p40 in $21 / 28$ metastases $(75 \%)$. 

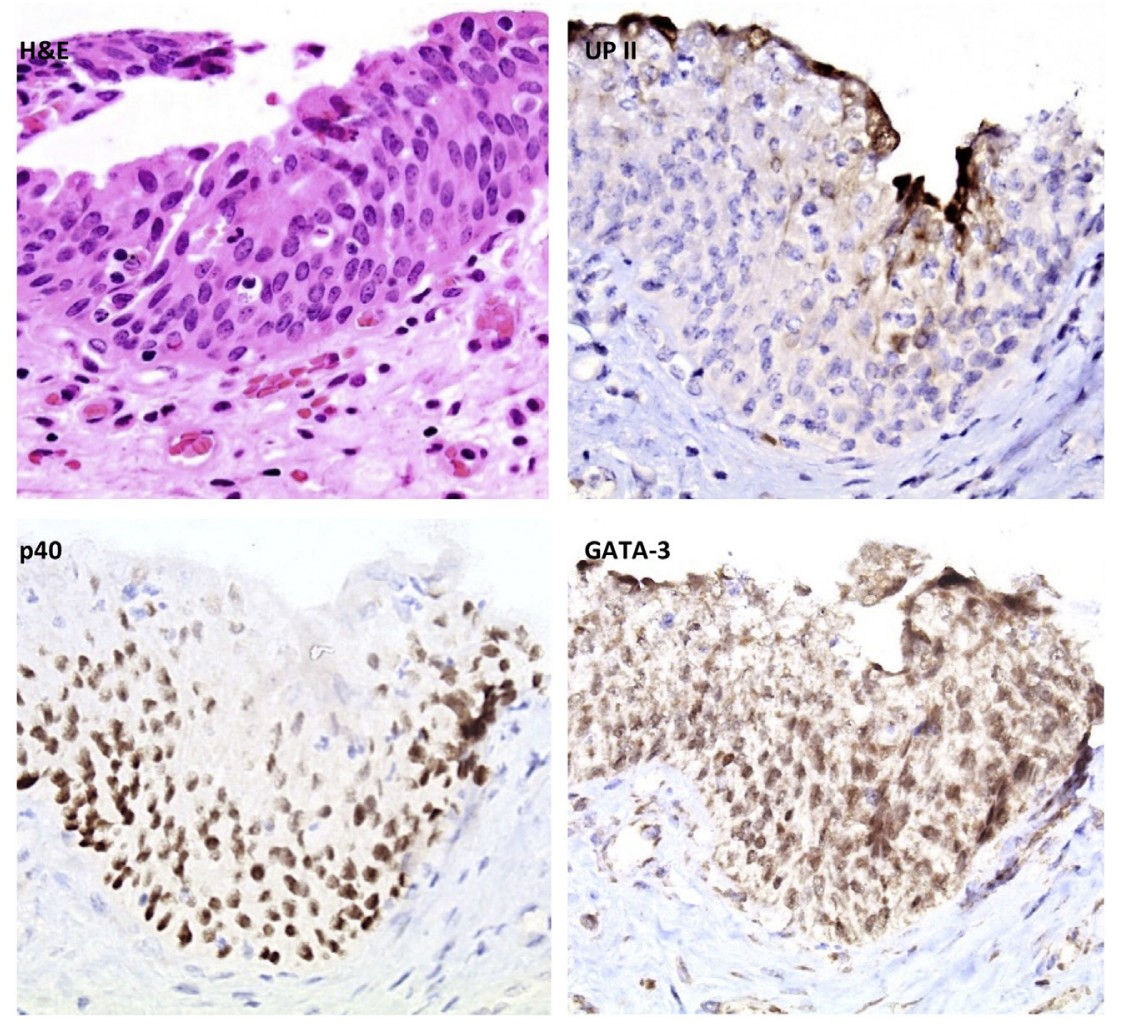

Fig. 1 Histology of normal urothelium stained by haematoxylin and eosin and immunohistochemistry: UPII, p40 and GATA-3.

\section{High p40 intensity in bladder $\mathrm{UCa}$ is associated with reduced overall survival}

Univariable analysis was performed to compare clinicopathological features and IHC parameters to recurrence-free survival and overall survival. As anticipated, patients with advanced age ( $\geq 75$ years), higher pT stage (pT3 and pT4 disease), and presence of lymph node metastasis were associated with reduced recurrence-free survival $(p=0.003$, $p=0.03$, and $p=0.001$, respectively; Logrank test) and overall survival $(p=0.004, p=0.02, \quad$ and $p=0.0007$, respectively; Logrank test) (Table 3). Multivariable analysis showed the presence of lymph node metastasis and age 75 years or greater showed reduced recurrence-free survival (HR 2.76; 95\% CI 1.57-4.83 and HR 2.02; 95\% CI 1.18-3.46, respectively) and reduced overall survival (HR 3.09; 95\% CI $1.72-5.54$ and $2.15 ; 95 \%$ CI 1.24-3.71), respectively (Table 4). Finally, we evaluated GATA-3, UPII and p40 as prognostic markers of recurrence-free and overall survival. Only strong p40 expression (3+ intensity) in the primary bladder UCa showed an independent association with reduced overall survival $(p=0.03)$ irrespective of lymph node status (Fig. 3).

\section{DISCUSSION}

Immunohistochemical analysis of urothelial carcinoma is of diagnostic value in several settings, such as the exclusion of secondary involvement of the bladder by carcinoma arising at another site or in the identification of the site of origin of metastatic lesions of unknown primary. Historically, many antibodies used in this context show lack of specificity, but maintain utility when used as a diagnostic panel. Such antibodies include high-molecular weight cytokeratins, cytokeratin 7, cytokeratin 20, p63, thrombomodulin and GATA-3. ${ }^{8}$ More specific antibodies have gained wider acceptance, including S100P, ${ }^{12}$ which is expressed in urothelial and pancreaticobiliary lesions, and uroplakin III (UPIII), which appears specific to urothelial origin. ${ }^{13}$ However, the lower sensitivity of both S100P and UPIII in advanced high-grade bladder cancer ${ }^{14-16}$ has prompted the development of new IHC markers. In this study, we assessed the diagnostic and prognostic application of the more recently developed uroplakin II and p40 antibodies in the setting of advanced and metastatic urothelial carcinoma and tested the additional value of GATA-3 incorporation into this panel.

A commercial UPII antibody has recently been developed by Biocare Medical and several studies have assessed its sensitivity relative to UPIII, as well as its usefulness as a diagnostic IHC marker together with GATA-3. ${ }^{1-5}$ The uroplakin proteins are highly conserved, transmembrane proteins that function as key components of the urothelial plaque. ${ }^{17-19}$ The relatively restrictive expression of these proteins to the urothelium has generated long-standing interest in diagnostic applications for urothelial carcinoma. 10,20 For example, the first primary antibody against UPII was developed in rabbits and targeted a conserved bovine UPII epitope in 1995; this antibody showed strong immunoreactivity against the umbrella cell layer of normal human urothelium and specificity for urothelial carcinoma versus carcinomas arising in other sites. ${ }^{10}$

The newly commercialised UPII antibody shows similar specificity, but with improved sensitivity over UPIII. UPII versus UPIII sensitivity in invasive urothelial carcinoma of the bladder was shown in one study on 16 cases to have a sensitivity of $63 \%$ versus $19 \%$ and in another study of 174 cases of varying grades and stages to have a sensitivity of 
Table 2 Association of pathological features, p40, UPII and GATA-3 expression with metastatic disease

\begin{tabular}{|c|c|c|c|}
\hline & No mets $(n=46)$ & Mets $(n=43)$ & $p^{\mathrm{a}}$ \\
\hline \multicolumn{4}{|l|}{ Pathological T-stage, $n(\%)$} \\
\hline 3,4 & $30(65 \%)$ & $38(88 \%)$ & 0.01 \\
\hline \multicolumn{4}{|l|}{ Neo- or adjuvant chemotherapy, $n(\%)$} \\
\hline No & $39(85 \%)$ & $15(38 \%)$ & \\
\hline Yes & $7(15 \%)$ & $25(62 \%)$ & 0.0001 \\
\hline \multicolumn{4}{|l|}{ Carcinoma in situ, $n(\%)$} \\
\hline No & $18(39 \%)$ & $24(56 \%)$ & \\
\hline Yes & $28(61 \%)$ & $19(44 \%)$ & 0.14 \\
\hline \multicolumn{4}{|l|}{ Positive margins, $n(\%)$} \\
\hline No & $34(74 \%)$ & $28(65 \%)$ & \\
\hline Yes & $12(26 \%)$ & $15(35 \%)$ & 0.49 \\
\hline p40 H-score, median (range) & $220(0-300)$ & $150(0-280)$ & 0.001 \\
\hline UPII H-score, median (range) & $90(0-300)$ & $160(0-300)$ & 0.007 \\
\hline GATA-3 H-score, median (range) & $240(10-300)$ & $250(0-300)$ & 0.35 \\
\hline
\end{tabular}

Mets, metastases; UPII, uroplakin II.

${ }^{a}$ Fisher's exact test for categorical factors, Wilcoxon rank sum test for measured factors.
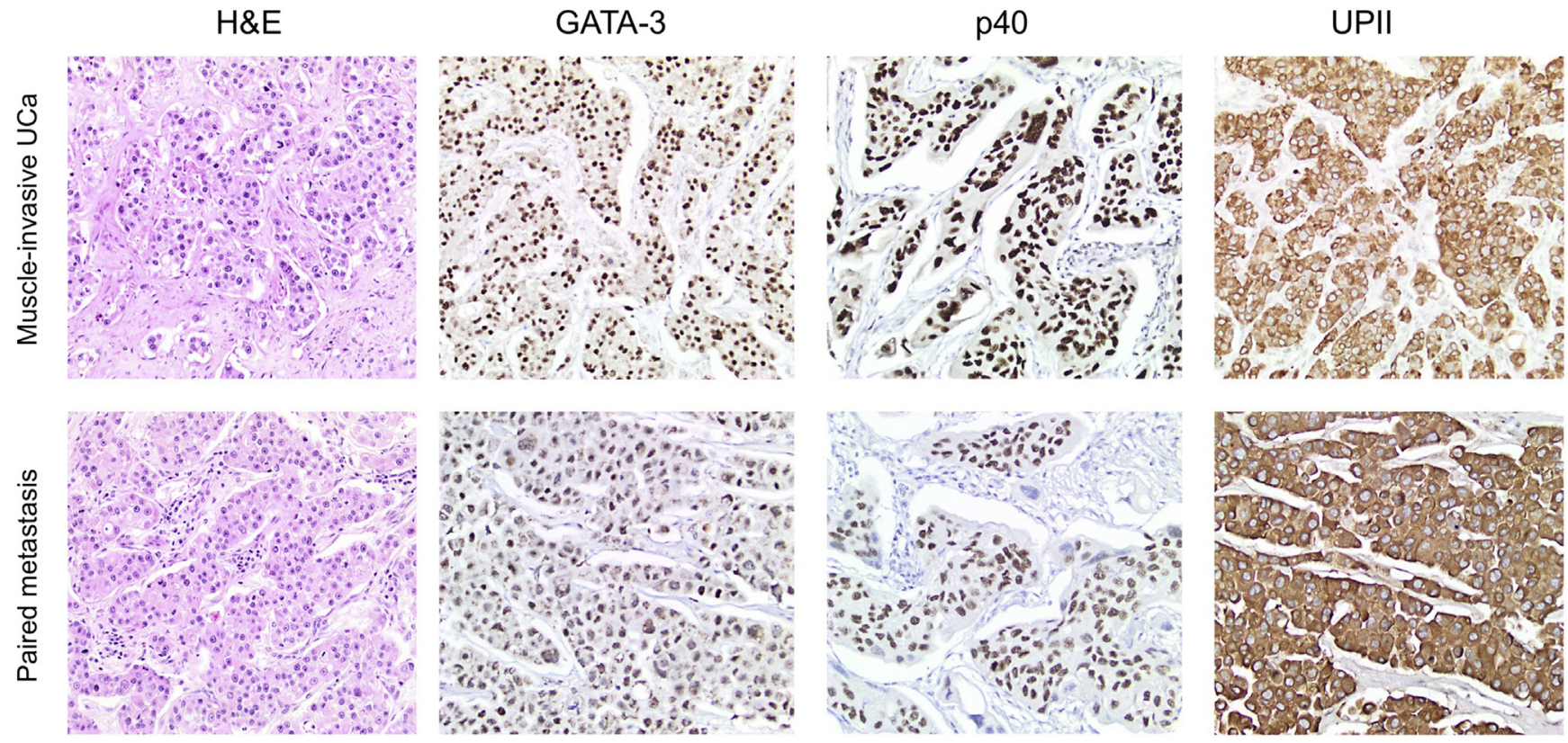

Fig. 2 Histology of invasive UCa using haematoxylin and eosin and immunohistochemical markers showing positive patterns (3+ score), where GATA-3 and p40 showed nuclear staining, and UPII showed diffused membranous and cytoplasmic expression.

$77 \%$ versus $54 \% .^{3,5}$ In non-invasive urothelial carcinoma that ranged from papillary urothelial neoplasm of low malignant potential (PUNLMP) to high-grade papillary urothelial carcinoma, UPII was reported to stain $>90 \%$ of cases, ${ }^{1}$ although a separate report from the upper tract appeared to have UPII retained primarily in umbrella cell layers of lower grade lesions. ${ }^{4}$ UPII also showed improved sensitivity over UPIII in nested, plasmacytoid, micropapillary and sarcomatoid UCa, although the number of plasmacytoid cases was somewhat limited in two studies. ${ }^{4,5}$ Conventional invasive UCa shows a range of immunoreactive frequency, with $44-78 \%$ of cases reported to be positive for UPII. ${ }^{1,4,5}$ However, in all comparisons, UPII outperformed UPIII in sensitivity.

In our study we focused primarily on the use of UPII in the diagnostically challenging setting of advanced bladder cancer and used paired lymph node metastases to assess changes in expression within an individual patient. When using any level of immunoreactivity, $80 \%$ of muscle-invasive UCa were positive for UPII, although this dropped to $74 \%$ when only moderate to intense immunoreactivity was considered. When used as a panel together with GATA-3 and p40, no primary bladder carcinoma or metastatic lesion was negative for all three markers. This suggests that a combination of GATA-3, UPII and p40 used together with more conventional, but less specific, immunohistochemical markers may be useful to identify urothelial origin in challenging cases and may be immunoreactive in a higher percentage of invasive UCa than previously reported. ${ }^{2}$ In contrast to both GATA-3 and p40, however, lymph node metastases were less likely to lose UPII expression, thus making addition of UPII to evaluation of metastases of unknown origin of potential value.

In conjunction with our analysis of UPII, we also incorporated another recently developed mouse monoclonal antibody against p40 $(\Delta \mathrm{Np} 63)$ by Biocare Medical $(\mathrm{BC} 28)$ that 
Table 3 Univariable analysis to compare clinicopathological features and patient outcomes

\begin{tabular}{|c|c|c|c|c|}
\hline \multirow[t]{2}{*}{ Factor } & \multicolumn{2}{|c|}{ Recurrence-free survival } & \multicolumn{2}{|c|}{ Overall survival } \\
\hline & All patients & $p^{\mathrm{a}}$ & All patients & $p^{\mathrm{a}}$ \\
\hline \multicolumn{5}{|l|}{ Gender } \\
\hline Male & 18.5 & & 21.6 & \\
\hline Female & 18.7 & 0.07 & 21.2 & 0.84 \\
\hline Age at diagnosis & & 0.04 & & 0.04 \\
\hline$\geq 75$ & 13.04 & 0.003 & 13.4 & 0.004 \\
\hline \multicolumn{5}{|l|}{ LN mets } \\
\hline No & 30.1 & & 42.6 & \\
\hline Yes & 12.2 & 0.001 & 13.4 & 0.0007 \\
\hline \multicolumn{5}{|c|}{ Pathological T-stage } \\
\hline 1,2 & 75.4 & & 75.4 & \\
\hline 3,4 & 15.4 & 0.03 & 18.3 & 0.02 \\
\hline \multicolumn{5}{|l|}{ CIS } \\
\hline No & 18.8 & & 21.6 & \\
\hline No & 21.5 & & 22.2 & \\
\hline Yes & 13.8 & 0.18 & 15.6 & 0.17 \\
\hline \multicolumn{5}{|l|}{$\mathrm{p} 40$} \\
\hline H-score & & 0.88 & & 0.62 \\
\hline \multicolumn{5}{|c|}{ Any level 3 staining } \\
\hline No & 30.2 & & 26.8 & \\
\hline Yes & 15.4 & 0.13 & $\begin{array}{l}20.0 \\
18.5\end{array}$ & 0.20 \\
\hline \multicolumn{5}{|l|}{ UPII } \\
\hline H-score & & 0.65 & & 0.65 \\
\hline \multicolumn{5}{|c|}{ Any UPII staining } \\
\hline No & 30.2 & & 40.5 & \\
\hline Yes & 17.2 & 0.62 & 20.6 & 0.38 \\
\hline \multicolumn{5}{|c|}{$>30 \%$ level $2 / 3$ staining } \\
\hline No & 17.2 & & 21.6 & \\
\hline Yes & 18.8 & 0.50 & 21.2 & 0.77 \\
\hline \multicolumn{5}{|l|}{ GATA-3 } \\
\hline $\mathrm{H}$-score & & 0.94 & & 0.76 \\
\hline
\end{tabular}

CIS, carcinoma in situ; LN, lymph node; Mets, metastases; UPII, uroplakin II.

${ }^{a}$ Log rank test.

has been reported to have lower variability than existing commercial antibodies. ${ }^{6}$ p40 represents a shorter isoform of the TP63 gene product that lacks the transactivation domain located within the $\mathrm{N}$-terminus region $(\Delta \mathrm{Np} 63) .{ }^{21}$ Studies on lung cancer specimens have shown that $\mathrm{p} 40$ expression was restricted to squamous cell carcinoma and was highly associated with p53 alterations. ${ }^{6,22-25}$ More recently, BC28 has shown immunoreactivity in $>80 \%$ of cases of skin cancer, head and neck squamous carcinoma and urothelial carcinoma, but an absence of expression in other cancers including prostatic adenocarcinoma and breast cancer. ${ }^{6}$ The extent of squamous differentiation in this UCa population was not described, although p63 expression and its p40 isoform likely share similar expression patterns in these tumour populations. Although the use of $\mathrm{p} 40$ as part of a diagnostic panel may be of value in $\mathrm{UCa}$, its solitary application in this context may be limited. In addition, use of this panel may also be of value in challenging diagnostic settings, such as the distinction of metastatic urothelial carcinoma from lung squamous cell carcinoma. $^{2}$

We tested the ability of GATA-3, UPII and p40 to predict recurrence-free and overall survival, which included progressive metastatic disease in 43 of 89 patients and death of disease in 55 of 89 patients. Given the frequent expression of both GATA-3 and UPII in this population, no significant difference in the expression patterns of these two markers using a cut-off of any versus no expression or low-level
$(0-1+)$ versus high-level $(2-3+)$ expression was seen. In contrast, high intensity p40 expression in the primary bladder UCa was associated with significantly shortened recurrencefree and overall survival. Although not significant in univariate analysis, $3+$ intensity of p40 staining was significant in multivariate analysis, which may be the result of unbalanced sample size, group variation or the presence of interaction. $^{26,27}$

Prior studies evaluating the prognostic role of p63 and p40 have yielded mixed results. Several studies have evaluated the prognostic role of p63 in bladder cancer patients, with p63 retention associated with either no effect or a worse prognosis. ${ }^{28-30}$ Expression of the p40 $(\Delta \mathrm{Np} 63)$ protein has been shown in two studies to confer a higher likelihood of disease progression in muscle-invasive bladder cancer, ${ }^{28,30}$ although one recent study using patients with pT1 disease showed that $\mathrm{p} 40$ expression loss is associated with a higher risk of progression. ${ }^{29}$ The data from our study suggest that p40 retention, at least in the context of advanced bladder cancer, was associated with increased likelihood of progression and death from disease, irrespective of lymph node status.

The oncogenic mechanism of p40 $(\Delta \mathrm{Np} 63)$ is not well defined, although a current study demonstrated a plausible explanation $^{31}$ and proposed that $\mathrm{p} 40$ is able to increase mRNA and protein levels of MTSS1 (Metastasis suppressor 1; Missing in Metastasis; MIM/BEG4), a protein involved in 
Table 4 Multivariable analysis to compare clinicopathological features and p40 IHC properties with recurrence-free and overall survival

Hazard ratio $(95 \% \mathrm{CI}) \quad p$

\begin{tabular}{lll}
\hline & & \\
Recurrence-free survival & $2.76(1.57-4.83)$ & 0.0004 \\
LN mets (No vs Yes) & $2.02(1.18-3.46)$ & 0.01 \\
Age $(<75$ vs $>75)$ & $1.93(1.07-3.46)$ & 0.03 \\
Any level 3 p40 staining (No vs Yes) & \\
Overall survival & $3.09(1.72-5.54)$ & 0.0002 \\
LN mets (No vs Yes) & $2.15(1.24-3.71)$ & 0.006 \\
Age (<75 vs $\geq 75)$ & $1.94(1.08-3.51)$ & 0.03 \\
Any level 3 p40 staining (No vs Yes) & & \\
\hline
\end{tabular}

CI, confidence interval; LN, lymph node; Mets, metastases. ${ }^{\mathrm{a}}$ Wald test.

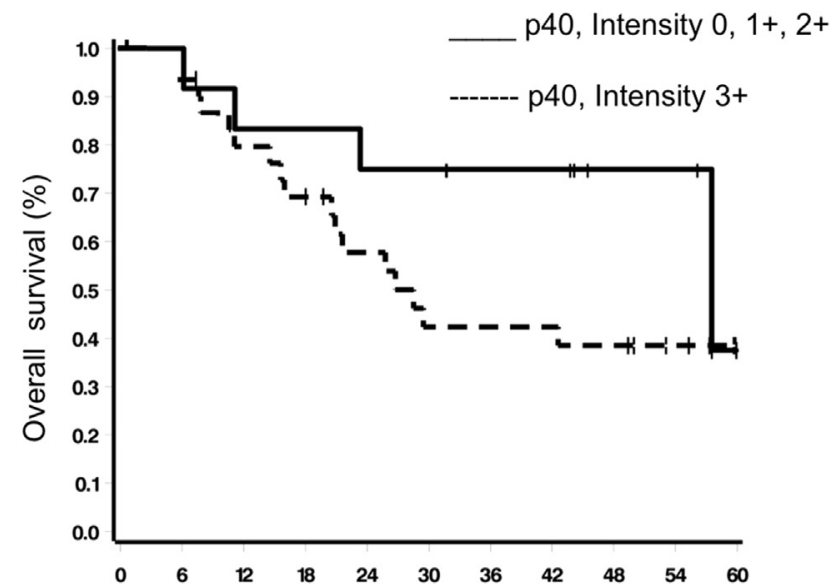

A

Follow-up (months)

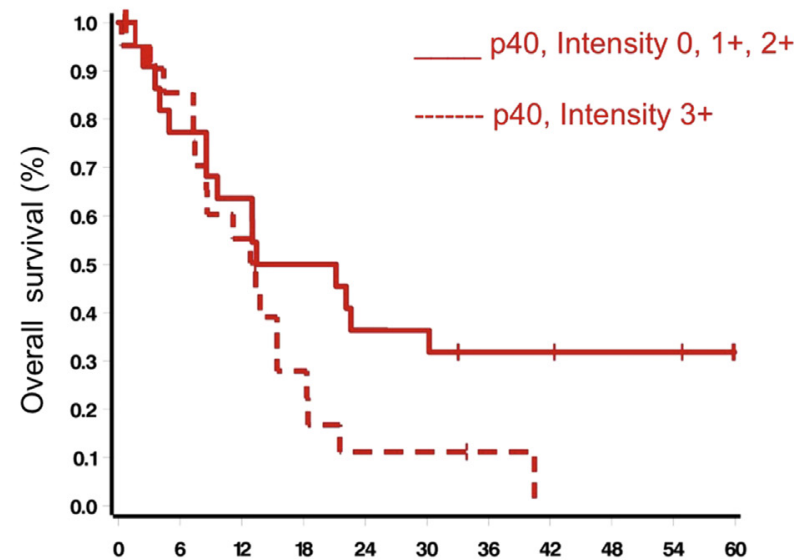

B

Follow-up (months)

Fig. 3 Reduced overall survival of patients with strong p40 expression (3+ intensity), irrespective of lymph node metastasis. (A) Patient with lymph node metastasis; (B) patient without lymph node metastasis.

promoting cell migration and invasion through reorganisation of the actin cytoskeleton. While in vitro pro-migratory ability was demonstrated only in breast cancer cell lines, the analysis of 138 bladder cancer samples also showed strong correlation between $\Delta \mathrm{Np} 63$ isoform and MTSS1 expression, suggesting that $\mathrm{p} 40 / \mathrm{MTSS} 1$ axis could also be involved in cell migration and invasion of bladder cancer. Another study suggests that retained and/or elevated p40 may be associated with a subset of aggressive urothelial carcinomas that maintain expression of the miR200 family and E-cadherin. ${ }^{28,32}$ Finally, p40 expression in UCa may be related to underlying molecular subtypes of bladder cancer, although this was not explored in our study. Specifically, the expression of basal cell markers such as p63, and likely p40, has been associated with an increased likelihood of aggressive disease. ${ }^{33}$ Future studies that evaluate the persistent expression of epithelial markers in advanced bladder cancer, and their relationship to the epithelial-mesenchymal transition process and therapeutic response, are required to address this question.

In conclusion, our study has shown that the newly developed UPII and p40 antibodies may be useful ancillary tools in muscle-invasive and metastatic urothelial carcinoma. Additional studies that evaluate a larger prospective patient population with muscle-invasive bladder cancer may be of value, especially in the context of the role of p40 in disease progression.

Conflicts of interest and sources of funding: The authors state there are no conflicts of interest to disclose.

Address for correspondence: Donna E. Hansel, MD PhD, UCSD Department of Pathology, 9500 Gilman Drive, MC 0612, La Jolla, CA 92093 USA. E-mail: dhansel@ucsd.edu

\section{References}

1. Tian W, Guner G, Miyamoto H, et al. Utility of uroplakin II expression as a marker of urothelial carcinoma. Hum Pathol 2015; 46: 58-64.

2. Hoang LL, Tacha D, Bremer RE, et al. Uroplakin II (UPII), GATA3, and p40 are highly sensitive markers for the differential diagnosis of invasive urothelial carcinoma. Appl Immunohistochem Mol Morphol 2015; 23: 711-6.

3. Hoang LL, Tacha DE, Qi W, et al. A newly developed uroplakin II antibody with increased sensitivity in urothelial carcinoma of the bladder. Arch Pathol Lab Med 2014; 138: 943-9.

4. Li W, Liang Y, Deavers MT, et al. Uroplakin II is a more sensitive immunohistochemical marker than uroplakin III in urothelial carcinoma and its variants. Am J Clin Pathol 2014; 142: 864-71.

5. Smith SC, Mohanty SK, Kunju LP, et al. Uroplakin II outperforms uroplakin III in diagnostically challenging settings. Histopathology 2014; 65: 132-8.

6. Tacha D, Bremer R, Haas T, et al. An immunohistochemical analysis of a newly developed, mouse monoclonal p40 (BC28) antibody in lung, bladder, skin, breast, prostate, and head and neck cancers. Arch Pathol Lab Med 2014: 138: 1358-64.

7. Gailey MP, Bellizzi AM. Immunohistochemistry for the novel markers glypican 3, PAX8, and p40 (DeltaNp63) in squamous cell and urothelial carcinoma. Am J Clin Pathol 2013; 140: 872-80.

8. Amin MB, Trpkov K, Lopez-Beltran A, et al. Best practices recommendations in the application of immunohistochemistry in the bladder lesions: report from the International Society of Urologic Pathology consensus conference. Am J Surg Pathol 2014; 38: e20-34.

9. Ohtsuka Y, Kawakami S, Fujii Y, et al. Loss of uroplakin III expression is associated with a poor prognosis in patients with urothelial carcinoma of the upper urinary tract. BJU Int 2006; 97: 1322-6.

10. Moll R, Wu XR, Lin JH, et al. Uroplakins, specific membrane proteins of urothelial umbrella cells, as histological markers of metastatic transitional cell carcinomas. Am J Pathol 1995; 147: 1383-97.

11. John T, Liu G, Tsao MS. Overview of molecular testing in non-smallcell lung cancer: mutational analysis, gene copy number, protein expression and other biomarkers of EGFR for the prediction of response to tyrosine kinase inhibitors. Oncogene 2009; 28(Suppl 1): S14-23.

12. Higgins JP, Kaygusuz G, Wang L, et al. Placental S100 (S100P) and GATA3: markers for transitional epithelium and urothelial carcinoma discovered by complementary DNA microarray. Am J Surg Pathol 2007; 31: 673-80. 
13. Kaufmann O, Volmerig J, Dietel M. Uroplakin III is a highly specific and moderately sensitive immunohistochemical marker for primary an metastatic urothelial carcinomas. Am J Clin Pathol 2000; 113: 683-7.

14. Chuang AY, DeMarzo AM, Veltri RW, et al. Immunohistochemica differentiation of high-grade prostate carcinoma from urothelial carcinoma. Am J Surg Pathol 2007; 31: 1246-55.

15. Parker DC, Folpe AL, Bell J, et al. Potential utility of uroplakin III, thrombomodulin, high molecular weight cytokeratin, and cytokeratin 20 in noninvasive, invasive, and metastatic urothelial (transitional cell) carcinomas. Am J Surg Pathol 2003; 27: 1-10.

16. Mhawech P, Uchida T, Pelte MF. Immunohistochemical profile of highgrade urothelial bladder carcinoma and prostate adenocarcinoma. Hum Pathol 2002; 33: 1136-40.

17. Yu J, Manabe M, Wu XR, et al. Uroplakin I: a 27-kD protein associated with the asymmetric unit membrane of mammalian urothelium. J Cell Biol 1990; 111: 1207-16.

18. Wu XR, Manabe M, Yu J, et al. Large scale purification and immunolocalization of bovine uroplakins I, II, and III. Molecular markers of urothelial differentiation. J Biol Chem 1990; 265: 19170-9.

19. Wu XR, Lin JH, Walz T, et al. Mammalian uroplakins. A group of highly conserved urothelial differentiation-related membrane proteins. J Biol Chem 1994; 269: 13716-24.

20. Olsburgh J, Harnden P, Weeks R, et al. Uroplakin gene expression in normal human tissues and locally advanced bladder cancer. $J$ Patho 2003; 199: 41-9.

21. Nobre AR, Albergaria A, Schmitt F. p40: a p63 isoform useful for lung cancer diagnosis - a review of the physiological and pathological role of p63. Acta Cytol 2013; 57: 1-8.

22. Bishop JA, Teruya-Feldstein J, Westra WH, et al. p40 (DeltaNp63) is superior to p63 for the diagnosis of pulmonary squamous cell carcinoma. Mod Pathol 2012; 25: 405-15.

23. Pelosi G, Fabbri A, Bianchi F, et al. DeltaNp63 (p40) and thyroid transcription factor-1 immunoreactivity on small biopsies or cellblocks for typing non-small cell lung cancer: a novel two-hit, sparing-material approach. J Thorac Oncol 2012; 7: 281-90.

24. Nonaka D. A study of DeltaNp63 expression in lung non-small cell carcinomas. Am J Surg Pathol 2012; 36: 895-9.

25. Brown AF, Sirohi D, Fukuoka J, et al. Tissue-preserving antibody cocktails to differentiate primary squamous cell carcinoma, adenocarcinoma, and small cell carcinoma of lung. Arch Pathol Lab Med 2013; 137: $1274-81$.

26. Lo SK, Li IT, Tsou TS, et al. Non-significant in univariate but signifcant in multivariate analysis: a discussion with examples. (Chinese.) Changgeng Yi Xue Za Zhi 1995; 18: 95-101.

27. Trikalinos TA, Hoaglin DC, Schmid CH. An empirical comparison of univariate and multivariate meta-analyses for categorical outcomes. Stat Med 2014; 33: 1441-59.

28. Choi W, Shah JB, Tran M, et al. p63 expression defines a lethal subset of muscle-invasive bladder cancers. PloS One 2012; 7: e30206.

29. Gaya JM, Lopez-Martinez JM, Karni-Schmidt O, et al. DeltaNp63 expression is a protective factor of progression in clinical high grade $\mathrm{T} 1$ bladder cancer. J Urol 2015; 193: 1144-50.

30. Karni-Schmidt O, Castillo-Martin M, Shen TH, et al. Distinct expression profiles of p63 variants during urothelial development and bladder cancer progression. Am J Pathol 2011; 178: 1350-60.

31. Giacobbe A, Compagnone M, Bongiorno-Borbone L, et al p63 controls cell migration and invasion by transcriptional regulation of MTSS1. Oncogene 2016; 35: 1062-8.

32. Tran MN, Choi W, Wszolek MF, et al. The p63 protein isoform DeltaNp63alpha inhibits epithelial-mesenchymal transition in human bladder cancer cells: role of MIR-205. J Biol Chem 2013; 288 3275-88.

33. Choi W, Porten S, Kim S, et al. Identification of distinct basal and luminal subtypes of muscle-invasive bladder cancer with different sensitivities to frontline chemotherapy. Cancer Cell 2014; 25: 152-65. 